5 Thelin A. Hip joint arthrosis: an occupational disorder amongst farmers. Am J Ind Med 1990;18:339-43.

6 Croft P, Coggon D, Cruddas M, Cooper C. Osteoarthritis of the hip: an occupational disease in farmers. BMJ 1992;304:1269-72.

7 O'Brien T, Moran R, McGoldrick F. The aetiology of degenerative disease of the hip. Ir J Med Sci 1989;158:63-6.

8 Stecher RM. Heberden's nodes. Hereditary in hypertrophic arthritis of the finger joints. Am J Med Sci 1941;201:801-9.

9 Spector TD, Cicuttini FM, Baker J, Loughlin J, Hart DJ. Genetic influences on osteoarthritis in women: a twin study. BMJ 1996;312:940-4

10 Felson DT, Couropmitree N, Chaisson C, Hannan MT, Zhang Y, McAlindon TE, et al. Evidence for a Mendelian gene in a segregation analysis of generalised osteoarthritis. Arthritis Rheum 1998:41:1064-71.

11 Hirsch R, Lethbridge-Cejku M, Hanson R, Scott WW, Reichle R, Plato $\mathrm{CC}$, et al. Familial aggregation of osteoarthritis. Data from the Baltimore longitudinal study on aging. Arthritis Rheum 1998;41:1227-32.

12 Lindberg H. Prevalence of primary coxarthrosis in siblings of patients with primary coxarthrosis. Clin Orthop 1986;203:273-5

13 Chitnavis J, Sinsheimer J, Clipsham K, Loughlin J, Sykes B, Burge P, et al. Genetic influences in end-stage osteoarthritis. J Bone Joint Surg $(\mathrm{Br})$ 1997;79-B(4):660-4.

14 Fox K, Hochberg MC, Resnik C, Kenzora J, Hebel R, Zimmerman S, et al. Severity of radiographic findings in hip osteoarthritis associated with total hip arthroplasty. J Rheumatol 1996;23:693-7.

15 O’Reilly S, Doherty S, Johnson S, Muir K, Doherty M. Screening for hand osteoarthritis using a postal survey. Osteoarthritis Cartilage 1999;7:461-5.
16 Altman RD, Hochberg MC, Murphy WA, Wolfe F. Atlas of individua radiographic features in osteoarthritis. Osteoarthritis Cartilage 1995;3(suppl A):3-70.

17 Croft P, Cooper C, Wickham C, Coggon D. Defining osteoarthritis of the hip for epidemiologic studies. Am J Epidemiol 1990;132:514-22.

18 Cohen J. A coefficient of agreement for nominal scales. Educ Psychol Mea 1960;20:37-46

19 Bland J, Altman DG. Statistical methods for assessing agreement between two methods of clinical assessment. Lancet 1986;i:307-10.

20 Roach KE, Persky V, Miles T, Budiman-Mak E. Biomechanical aspects of occupation and osteoarthritis of the hip: a case-control study. J Rheumatol 1994;21:2334-40

21 Tepper S, Hochberg MC. Factors associated with hip osteoarthritis: data from the first national health and nutrition examination survey (NHANES-I). Am J Epidemiol 1993;137:1081-8.

22 Danielsson L. Incidence and prognosis of coxarthrosis. Acta Orthop Scand Suppl 1964;66:1-114

23 Jorring K. Osteoarthritis of the hip. Acta Orthop Scand 1980;51:523-30

24 Danielsson L, Lindberg H, Nilsson B. Prevalence of coxarthrosis. Clin Orthop 1984;191:110-5.

25 Smith R, Egger P, Coggon D, Cawley MID, Cooper C. Osteoarthritis of the hip joint and acetabular dysplasia in women. Ann Rheum Dis 1995;54:179-81.

(Accepted 15 August 2000)

\title{
Risk of gastrointestinal haemorrhage with long term use of aspirin: meta-analysis
}

\author{
Sheena Derry, Yoon Kong Loke
}

\begin{abstract}
Objectives To assess the incidence of gastrointestinal haemorrhage associated with long term aspirin therapy and to determine the effect of dose reduction and formulation on the incidence of such haemorrhage.

Design Meta-analysis of 24 randomised controlled trials (almost 66000 participants).

Intervention Aspirin compared with placebo or no treatment, for a minimum of one year.

Main outcome measures Incidence of gastrointestinal haemorrhage.

Results Gastrointestinal haemorrhage occurred in $2.47 \%$ of patients taking aspirin compared with $1.42 \%$ taking placebo (odds ratio 1.68; $95 \%$ confidence interval 1.51 to 1.88 ); the number needed to harm was 106 (82 to 140) based on an average of 28 months' therapy. At doses below $163 \mathrm{mg} /$ day, gastrointestinal haemorrhage occurred in $2.30 \%$ of patients taking aspirin compared with $1.45 \%$ taking placebo (1.59; 1.40 to 1.81$)$. Meta-regression showed no relation between gastrointestinal haemorrhage and dose. For modified release formulations of aspirin the odds ratio was 1.93 (1.15 to 3.23).

Conclusions Long term therapy with aspirin is associated with a significant increase in the incidence of gastrointestinal haemorrhage. No evidence exists that reducing the dose or using modified release formulations would reduce the incidence of gastrointestinal haemorrhage.
\end{abstract}

\section{Introduction}

The use of aspirin in the prevention of cardiovascular disease is now well established; an estimated 50 million Americans have started taking aspirin over the past two decades. ${ }^{1}$ However, aspirin causes haemorrhagic complications. A systematic review in 1993 showed that the risk of gastrointestinal haemorrhage was significantly increased by long term aspirin. ${ }^{2}$ Only four of the 21 trials included in that review, however, used doses below $300 \mathrm{mg}$ a day. Since then, new data have become available from eight studies involving 24964 patients taking aspirin doses of 50-162.5 $\mathrm{mg}$ a day.

Recent trends towards the use of lower doses have been driven by the belief that these offer a better safety profile while retaining equivalent therapeutic efficacy. Is there evidence that the risk of gastrointestinal haemorrhage is substantially reduced at lower doses, and if so, by how much? Expensive "modified release" formulations have been developed in an attempt to reduce the likelihood of adverse gastrointestinal effects. What is the evidence that they do so?

We reviewed the safety of aspirin, studying the effect of dose and formulation and incorporating the new data from the eight studies mentioned above.

\section{Methods}

The review was conducted using a defined protocol.

\section{Inclusion criteria}

Studies-We included reports if they were full journal publications of randomised controlled trials of aspirin used as an antiplatelet agent. Trials were excluded if the term "randomised" was not specifically mentioned in the report or if the investigators clearly used non-random allocation, such as by date of birth. Abstracts, review articles, case reports, clinical observations, and unpublished data were not included. Trials with fewer than 50 patients in each arm were not included in the analysis because they were unlikely to be able to detect uncommon or rare adverse effects."
Editorial by Tramèr

Department of Clinical

Pharmacology,

University of

Oxford, Radcliffe

Infirmary, Oxford

OX2 6HE

Sheena Derry

research assistant

Yoon Kong Loke clinical lecturer in

clinical pharmacology

Correspondence to:

Y K Loke

yoon.loke@clinpharm ox.ac.uk

BMJ 2000;321:1183-7

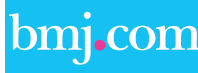

A further table and figure plus the references for the included studies appear on the BMJ's website 
We did not include studies designed to assess the effects of aspirin in special groups-such as pregnant women, children, and patients with pre-existing platelet disorders.

Intervention -We included trials in which patients in one treatment arm were allocated to oral aspirin alone and patients in the control arm to either placebo or no treatment, provided that the scheduled duration of treatment was at least 12 months. Crossover studies and those in which aspirin was used in conjunction with other antiplatelet agents or anticoagulants were excluded. Trials that compared aspirin at different doses or with other antiplatelet agents or anticoagulants, without a placebo or "no treatment" control arm, were also excluded.

Outcome measures-Only trials that provided numerical data on all gastrointestinal haemorrhages in both the treated and the control group were included. If specific terms were used to describe bleeding complications, we accepted data on patients with "haematemesis" or "melaena," or with both of these, but not with "proctorrhagia." We did not use data from trials that reported only on selected categories of gastrointestinal haemorrhage-for example, major bleeds and patients needing admission to hospital or blood transfusion-as trials varied considerably in their definition and reporting of such categories. ${ }^{4}$

\section{Search strategy}

The search was performed separately by each author. Relevant trials were identified through a combination of electronic searching and manual checking of reference lists from previous review papers and retrieved trials. We applied no language restrictions.

We concentrated specifically on selecting trials from a detailed list of over 200 antiplatelet studies identified in a systematic review that included trials published up to $1993 .{ }^{5}$ In view of the comprehensive search strategies used in that review, we chose not to repeat an electronic search for most of this period but carried out a search of Medline and Embase for 1990-9 to identify new trials and to provide a three year overlap. We found no discrepancy between the two lists of the trials identified in the overlap period.

We used a sensitive search string for randomised controlled trials, based on that of the Cochrane Collaboration, ${ }^{6}$ in combination with the following free text terms: "aspirin" or "acetylsalicylic*" or "salicylic*". This gave a yield of over 7000 hits on Medline and over 10000 hits on Embase for 1990-9. We made no attempt to limit the yield by introducing terms for adverse effects or haemorrhage as we knew that this strategy would lead to the loss of relevant trials. ${ }^{7}$

\section{Appraisal of study quality and data abstraction}

We did not anonymise the reports before assessment. All potentially relevant studies were checked independently by both reviewers to determine eligibility for inclusion and to extract data. A list of trials which were excluded is available from the authors.

We sought information on participants, blinding, type of control, assessment of compliance, and duration of treatment and follow up, in addition to data on the numbers of participants with and without gastrointestinal haemorrhage. We also recorded the formulation of aspirin used-modified release or standard. Any discrepancies were resolved by discussion.

Statistical analysis

Pooled odds ratios and heterogeneity were analysed using the RevMan program, version 4.04, with Meta

Characteristics of studies included in meta-analysis

\begin{tabular}{|c|c|c|c|c|c|}
\hline Study & Year & Aspirin formulation & Dose (mg/day) & $\begin{array}{l}\text { Average treatment } \\
\text { duration (months) }\end{array}$ & Indication for aspirin therapy \\
\hline Diener et al ${ }^{\mathrm{w} 1} \mathrm{w}_{2}$ & 1996 & Unspecified & 50 & 24 & Transient ischaemic attack or stroke \\
\hline Hansson et al ${ }^{\text {w3 }}$ & 1998 & Standard & 75 & 45 & Hypertension \\
\hline Petersen et al ${ }^{\mathrm{w} 4}$ & 1989 & Unspecified & 75 & 24 & Atrial fibrillation \\
\hline$\overline{\mathrm{SALT}} \mathrm{w}^{\mathrm{w}}$ & 1991 & Standard & 75 & 32 & Transient ischaemic attack or stroke \\
\hline$\overline{\text { TPT }}{ }^{\mathrm{w} 6}$ & 1998 & Modified release & 75 & 81 & Cardiovascular risk factors \\
\hline Wallentin $^{\text {w7 }}$ & 1991 & Unspecified & 75 & 12 & Unstable angina \\
\hline Silagy et al ${ }^{\mathrm{w8}}$ & 1993 & Standard & 100 & 12 & Primary prevention \\
\hline USPHS $^{w 9}$ & 1989 & Standard & 162.5 & 60 & Primary prevention \\
\hline $\mathrm{EAFT}^{\text {w10 }}$ & 1993 & Unspecified & 300 & 28 & Transient ischaemic attack or stroke \\
\hline Elwood et alw11 & 1974 & Standard & 300 & 12 & Myocardial infarction \\
\hline UK-TIA ${ }^{\text {w12 }}$ & 1991 & Various & 300,1200 & 48 & Transient ischaemic attack \\
\hline Gavaghan et alw13 & 1991 & Standard & 324 & 12 & Coronary artery bypass grafting \\
\hline Olivotto et al ${ }^{\mathrm{w} 14}$ & 1996 & Modified release & 325 & 12 & Breast cancer \\
\hline Elwood et alw15 & 1979 & Standard & 900 & 12 & Myocardial infarction \\
\hline$\overline{\mathrm{CDPA}^{\mathrm{w16}}}$ & 1976 & Unspecified & 972 & 22 & Myocardial infarction \\
\hline PARIS ${ }^{\text {w17 }}$ & 1980 & Unspecified & 972 & 41 & Myocardial infarction \\
\hline Hess et alw18 & 1985 & Unspecified & 990 & 24 & Peripheral vascular disease \\
\hline$\overline{\text { AMIS }^{\text {w19 }}}$ & 1980 & Standard & 1000 & 36 & Myocardial infarction \\
\hline Breddin et al ${ }^{\mathrm{w} 20}$ & 1980 & Modified release & 1000 & 24 & Myocardial infarction \\
\hline $\operatorname{CCSG}^{\text {W21 }}$ & 1978 & Standard & 1300 & 26 & Transient ischaemic attack \\
\hline Fields et al ${ }^{\mathrm{w} 22}$ & 1977 & Standard & 1300 & 24 & Transient ischaemic attack \\
\hline Fields et al ${ }^{\mathrm{w} 23}$ & 1978 & Standard & 1300 & 24 & Transient ischaemic attack \\
\hline Brittonw24 & 1987 & Modified release & 1500 & 24 & Stroke \\
\hline Ehresman et al ${ }^{\mathrm{w} 25}$ & 1977 & Modified release & 1500 & 12 & Peripheral vascular disease \\
\hline
\end{tabular}

SALT=Swedish aspirin low dose trial; TPT=thrombosis prevention trial; USPHS=US physicians health study; EAFT=European atrial fibrillation trial; UK-TIA=UK transient ischaemic attack aspirin trial; CDPA=coronary drug project aspirin study; PARIS=persantine-aspirin reinfarction study; AMIS=aspirin myocardial infarction study; CCSG=Canadian Cooperative Study Group. 


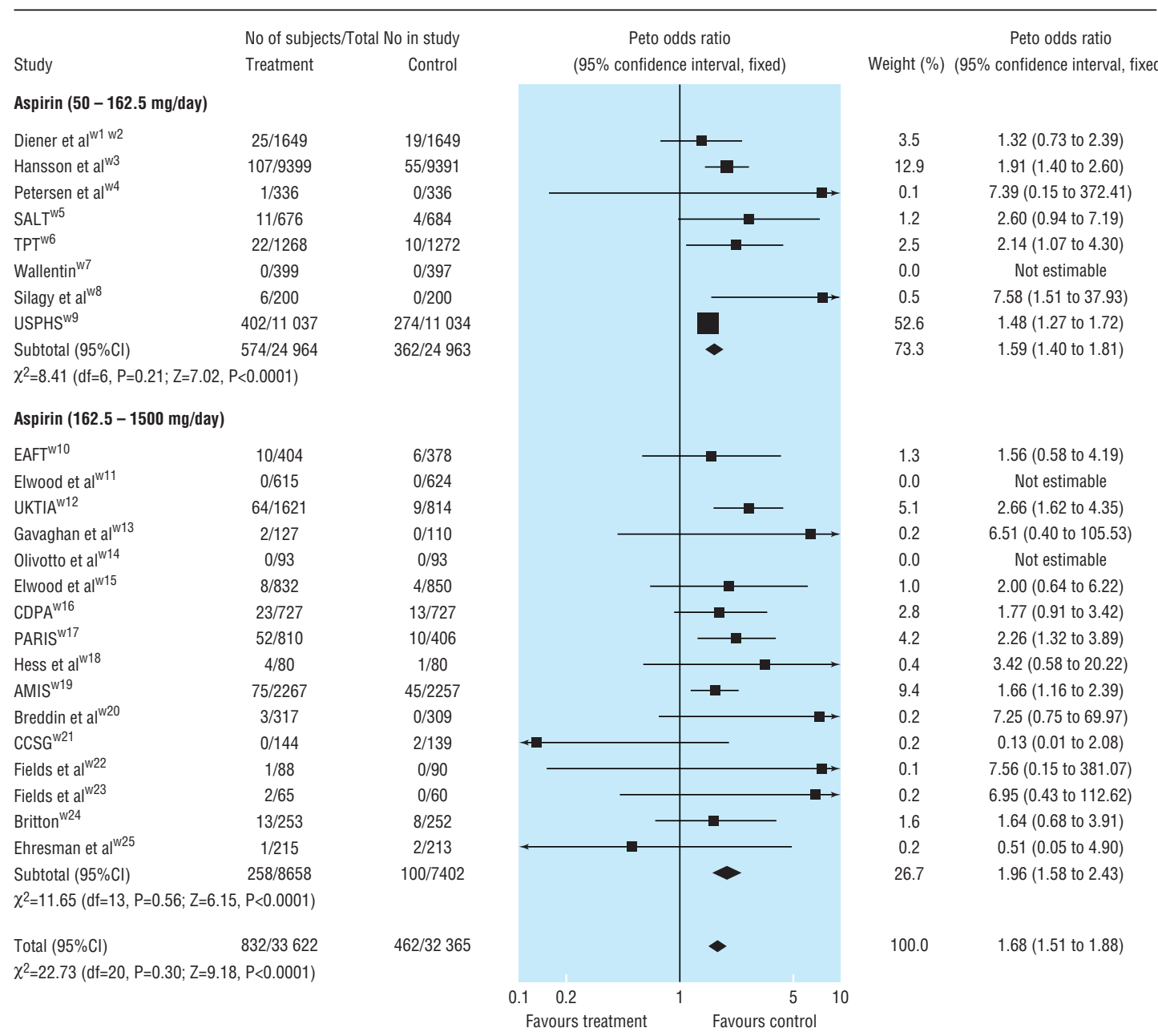

Fig 1 Peto odds ratio for gastrointestinal haemorrhage with aspirin. SALT=Swedish aspirin low dose trial; TPT=thrombosis prevention trial; USPHS=US physicians health study; EAFT=European atrial fibrillation trial; UK-TIA=UK transient ischaemic attack aspirin trial; $C D P A=c o r o n a r y$ drug project aspirin study; PARIS=persantine-aspirin reinfarction study; AMIS=aspirin myocardial infarction study; CCSG=Canadian Cooperative Study Group

View 4.0 and MetaView 3.01. We used the Peto fixed effects model to calculate the pooled odds ratios as this is the most appropriate model for rare events. ${ }^{8}$ Closely similar results were obtained with the random effects model. Meta-regression is a technique used to assess statistically whether specific factors (covariates) influence the magnitude of effect across studies. Random effects meta-regression was performed with the STATA "metareg" command. ${ }^{9}$

The number needed to harm (with 95\% confidence intervals) was calculated by applying the calculated odds ratio to the pooled control event rate. In our review the number needed to harm is the estimated number of patients who need to be treated with aspirin-rather than with placebo or no treatment-for one additional patient to be harmed by a gastrointestinal haemorrhage.

\section{Results}

We identified 24 randomised controlled trials of aspirin that fulfilled our inclusion criteria (table). (A further table and the references for the 24 trials $^{\mathrm{w1-w25}}$ are on the $B M j$ s website.) The 65987 participants were predominantly male $(74 \%)$ and middle aged. Doses of aspirin used were $50-1500 \mathrm{mg} /$ day for a mean duration of 28 months. Indications for aspirin extended from primary prevention in "healthy" individuals to secondary prophylaxis after stroke. In all trials patients were excluded if they had a history of peptic ulcer, previous gastrointestinal haemorrhage, or any other contraindication to aspirin.

All 24 trials were double blind and placebo controlled. Fourteen trials had sufficient data to suggest that adequate concealment of allocation had taken place; in the other trials this was unclear. The number of patients lost to follow up was reported in 16 trials, only one of which failed to achieve over $90 \%$ follow up.

Figure 1 shows the results of our meta-analysis. Overall, gastrointestinal haemorrhage occurred in $2.47 \%$ of the patients taking aspirin compared with $1.42 \%$ of those taking placebo. The pooled odds ratio for gastrointestinal haemorrhage with aspirin was $1.68(95 \%$ confidence interval 1.51 to 1.88 ; $\mathrm{P}<0.0001$ ), and the number needed to harm based on an average of 28 months of aspirin was 106 (82 to 


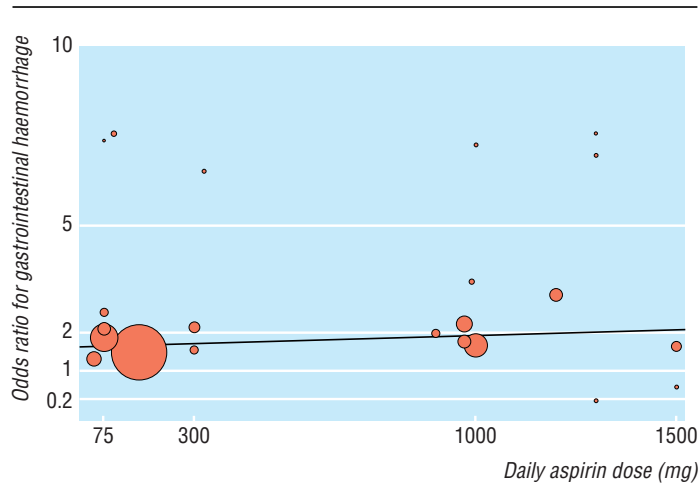

Fig 2 Meta-regression of Peto odds ratio for gastrointestinal haemorrhage against dose of aspirin (size of circle is proportional to size of trial)

140). We found no evidence of significant heterogeneity between the trials.

Current clinical practice favours the use of lower doses of aspirin, and to increase the relevance of our findings we analysed separately the eight trials that used doses of $50-162.5 \mathrm{mg}$ /day in 49927 participants. Gastrointestinal haemorrhage occurred in $2.30 \%$ of those taking aspirin compared with $1.45 \%$ taking placebo. Even at these lower doses, aspirin was associated with a significantly increased rate of gastrointestinal haemorrhage compared with placebo, with a pooled odds ratio of 1.59 (1.40 to $1.81 ; \mathrm{P}<0.0001)$.

We performed meta-regression to test for a linear relation between daily dose of aspirin and risk of gastrointestinal haemorrhage (fig 2 ). The analysis gave a pooled odds ratio of 1.015 (0.984 to 1.047$)$ per $100 \mathrm{mg}$ dose reduction, with an estimated relative reduction in the incidence of gastrointestinal haemorrhage of $1.5 \%$ per $100 \mathrm{mg}$ reduction of dose, but this was not significant $(\mathrm{P}=0.3)$.

\section{What is already known on this topic}

Aspirin is widely used to prevent and treat cardiovascular disease but carries an increased risk of gastrointestinal haemorrhage

Despite the lack of concrete evidence, lower doses and modified release formulations of aspirin have been used in the hope of reducing this risk

Data from several trials of low dose aspirin have become available recently

\section{What this study adds}

About 1 in 100 patients taking aspirin over a 28 month period will experience a gastrointestinal haemorrhage

No evidence exists that dose reduction or the use of modified release formulations significantly lowers the risk of gastrointestinal haemorrhage

Patients and doctors need to consider the trade-off between the benefits and harms of long term treatment with aspirin
Two of the trials ${ }^{\text {w3 }}{ }^{\text {w9 }}$ were much larger than the others, and to be sure that neither unduly influenced the results, we performed a sensitivity analysis. Omitting either or both trials from the meta-analysis did not significantly change our findings. Only five trials, with 4298 participants, specifically stated that modified release formulations of aspirin were used with daily doses of 75-1500 mg. ${ }^{\text {w6 w14 w20 w24 w25 }}$ The odds ratio for gastrointestinal haemorrhage in these five trials was 1.93 (1.15 to 3.23 ).

\section{Discussion}

Long term aspirin therapy, even at a low dose, carries a risk of gastrointestinal haemorrhage, with a number needed to harm per year of 248. Although it would be preferable, for the purposes of comparison, to have a number needed to harm and a number needed to treat from the same trial or review, this may not always be feasible. It is possible, however, to obtain some idea of the trade-off between benefit and harm when using aspirin in patients with different cardiovascular risk levels by weighing up the pooled estimate of the number needed to harm against the number needed to treat from individual trials.

In the secondary prevention of stroke, for example, the number needed to treat per year with aspirin to prevent a further event was $106 .{ }^{\mathrm{w} 5}$ This means that if aspirin was used in patients with stroke who had similar baseline risks to those above, at least two recurrent strokes could be prevented at the cost of one gastrointestinal haemorrhage. The benefits of aspirin are less marked, however, in primary prevention of myocardial infarction-in the US physicians study, the number needed to treat per year was $555,{ }^{\mathrm{w} 9}$ whereas the number needed to treat per year in hypertensive patients was 794. ${ }^{\mathrm{w} 3}$ Aspirin use in primary prevention could, depending on the baseline risks of the patients, cause two or three gastrointestinal haemorrhages for each myocardial infarction prevented.

As there are relatively few deaths after gastrointestinal haemorrhage (estimated death rate $12 \%{ }^{10}$ ) compared with myocardial infarction, such a trade-off may be considered worth while. Doctors and patients involved in making decisions about aspirin therapy need to consider carefully whether the inconvenience of long term therapy and the associated risk of gastrointestinal haemorrhage are outweighed by the potential cardiovascular benefits. This is particularly so for those at low absolute risk of cardiovascular events (with correspondingly high numbers needed to treat), in whom the likelihood of harm is greater than that of therapeutic benefit (as shown in the example above for hypertensive patients).

Although doctors have hoped that changes in the dose or formulation of aspirin might reduce the problem of gastrointestinal haemorrhage, the results of this meta-analysis do not suggest that either approach offers clear benefits. Our findings are supported by those of two recent case-control studies, in which aspirin increased the risk of gastrointestinal haemorrhage, despite the use of low dose or modified release formulations. ${ }^{11} 12$ The results of our meta-regression are compatible with those of a large Dutch trial of transient ischaemic attack in which no significant difference in 
the rate of gastrointestinal haemorrhage was found between two different doses of aspirin. ${ }^{13}$ In this study, aspirin was efficacious at a dose of $30 \mathrm{mg}$ a day, but a threshold dose for either the therapeutic or adverse effects of aspirin has yet to be established, and further attempts at dosage reduction might compromise therapeutic efficacy before adverse effects are eliminated completely.

Insufficient evidence exists to support the view that modified release formulations are safer, in terms of gastrointestinal haemorrhage, than standard formulations. Here we have studied the effect of dose and formulation on the incidence of gastrointestinal haemorrhage only; it may be that other symptomatic gastrointestinal adverse effects, such as nausea and epigastric pain, can be significantly reduced. ${ }^{13}$

The incidence of gastrointestinal haemorrhage with aspirin is relatively low, and to avoid factors that could have led us to underestimate the risk, we set inclusion and exclusion criteria such that only trials of a certain quality, with adequate numbers and follow up, would be selected. Although there is some asymmetry in the funnel plot (see figure on $B M / \mathrm{s}$ website), suggesting the possibility of selection bias, adjustment for the likely effect of bias using "trim and fill" gave a pooled odds ratio of 1.62 , which is only a slight change from our estimate of $1.68 .{ }^{14}$ Our meta-analysis seems reasonably robust to the asymmetry observed in the funnel plot.

We believe that the findings of our study are relevant to everyday practice. No significant heterogeneity was found, even though the studies we analysed encompassed a broad selection of patients with varying clinical indications. All the trials excluded patients at increased risk of gastrointestinal haemorrhage or with aspirin intolerance, but this is consistent with current advice on the use of aspirin and does not invalidate the relevance of our findings. Nevertheless, aspirin is available over the counter, and the risk of gastrointestinal haemorrhage could be higher in patients who take it without consulting a doctor.

We thank Jon Deeks for encouragement and statistical support, particularly with the meta-regression; Alex Sutton for helping with the funnel plot; and Jeff Aronson for help with the manuscript.

Contributors: YKL conceptualised the review, developed the protocol, provided clinical interpretation of the trials, abstracted data, and undertook most of the statistical analyses. SD contributed to the development of the protocol, abstracted data, and prepared the manuscript. Both authors will act as guarantors for the paper.

Funding: SD was supported by a grant from the Sir Jules Thorne Trust.

Competing interests: None declared.

1 Bayer Pharmaceuticals. Facts about aspirin. www.wonderdrug.com/press/ factsheets/aspirin_fact_sheet.pdf (accessed 28 July 2000).

2 Roderick PJ, Wilkes HC, Meade TW. The gastrointestinal toxicity of aspirin: an overview of randomised controlled trials. $\mathrm{Br} \mathrm{J}$ Clin Pharmacol 1993;35:219-26.

3 Eypasch E, Lefering R, Kum CK, Troidl H. Probability of adverse events that have not yet occurred: a statistical reminder. BMJ 1995;311: $619-20$.

4 Zanchetti A, Hansson L. Risk of major gastrointestinal bleeding with aspirin. Lancet 1999;353:148-50.

5 Collaborative overview of randomised trials of antiplatelet therapy-I Prevention of death, myocardial infarction, and stroke by prolonged antiplatelet therapy in various categories of patients. Antiplatelet Trialists' Collaboration. BMJ 1994;308:81-106.

6 Clarke M, Oxman AD, eds. Optimal search strategies for RCTs. Cochrane reviewers' handbook 4.0 (appendix 5c). In: Review Manager (RevMan) [computer program]. Version 4.0. Oxford: Cochrane Collaboration, 1999

7 Loke YK, Edwards J, Derry S. Conventional search strategies cannot easily identify those trials of drug therapy which provide quantitative adverse effects data [abstract]. Proceedings of the VII Cochrane Colloquium, Rome 1999. www.clinpharm.ox.ac.uk/SearchStrategy.htm (accessed 28 July 2000).

8 Deeks JJ, Bradburn MJ, Localio R, Berlin J. Much ado about nothing: meta-analysis for rare events [abstract]. Proceedings of 2nd symposium on systematic reviews: beyond the basics, Oxford 1999. www.ihs.ox.ac.uk/csm/ talks.html\#p23 (accessed 28 July 2000).

9 Sharp S. she 23: meta-analysis regression. Stata Technical Bulletin 1998;42:16-22.

10 Tramer MR, Moore RA, Reynolds DJM, McQuay HJ. Quantitative estimation of rare adverse events which follow a biological progression: a new model applied to chronic NSAID use. Pain 2000;85:169-82.

11 Weil J, Colin-Jones D, Langman M, Lawson D, Logan R, Murphy M, et al. Prophylactic aspirin and risk of peptic ulcer bleeding. BMJ 1995;310:827 30.

12 Kelly JP, Kaufman DW, Jurgelon JM, Sheehan J, Koff RS, Shapiro S. Risk of aspirin-associated major upper-gastrointestinal bleeding with entericcoated or buffered product. Lancet 1996;348:1413-6.

13 Dutch TIA Trial Study Group. A comparison of two doses of aspirin (30 $\mathrm{mg}$ vs $283 \mathrm{mg}$ a day) in patients after a transient ischemic attack or minot ischemic stroke. N Engl J Med 1991;325:1261-6.

14 Duval S, Tweedie R. Nonparametric "trim and fill" method for accounting for publication bias in meta-analysis. J Am Stat Assoc 2000;95:89-98.

(Accepted 15 August 2000)

\title{
Wet combing versus traditional scalp inspection to detect head lice in schoolchildren: observational study
}

\author{
Jan De Maeseneer, Ineke Blokland, Sara Willems, Robert Vander Stichele, Filip Meersschaut
}

Lice infestation is a problem in local communities, probably because reservoirs remain undetected. Wet combing (combing systematically through wet, well conditioned hair with a fine toothed comb) has been presented as a cheap, ecological, self sufficient, and feasible technique for diagnosis and treatment of head lice. ${ }^{1-3}$ Compared with traditional scalp inspection it uses five elements to make living lice more visible, to better distinguish them from dandruff, and to assess the maturity of the infestation: water, conditioner, a fine toothed comb, a systematic sweep of the scalp, and a magnifying glass $(10 \times)$. However, its efficacy as a diagnostic tool and as a therapeutic intervention has not been proved; hence it is not evidence based.

\section{Subjects, methods, and results}

We did an observational study comparing detection of head lice using traditional scalp inspection and wet combing. After ethical approval had been obtained, all 260 pupils, aged 2-12 years, of a primary school in a socially deprived urban area in Ghent, Belgium, were invited for a screening test during a three day campaign to detect head lice in November and
Department of General Practice and Primary Health Care, Ghent University, 1K3 UZ, B-9000 Ghent, Belgium Jan De Maeseneer professor Sara Willems researcher continued over BMJ 2000;321:1187-8 